Journal of Theoretical and Applied Mechanics, Sofia, Vol. 47 No. 1 (2017) pp. 69-84

\title{
THERMAL INSTABILITY IN A LAYER OF COUPLE STRESS NANOFLUID SATURATED POROUS MEDIUM
}

\author{
RAMESH CHAND $^{1 *}$, G. C. RANA ${ }^{2}$, DHANANJAY YADAV ${ }^{3}$ \\ ${ }^{1}$ Department of Mathematics, Government Arya Degree College Nurpur, \\ Himachal Pradesh, India \\ ${ }^{2}$ Department of Mathematics, NSCM Government College Hamirpur, \\ Himachal Pradesh, India \\ ${ }^{3}$ School of Mechanical Engineering, Yonsei University Seoul, South Korea
}

[Received 14 July 2015. Accepted 20 March 2017]

\begin{abstract}
Thermal instability in a horizontal layer of Couple-stress nanofluid in a porous medium is investigated. Darcy model is used for porous medium. The model used for nanofluid incorporates the effect of Brownian diffusion and thermophoresis. The flux of volume fraction of nanoparticle is taken to be zero on the isothermal boundaries. Normal mode analysis and perturbation method is employed to solve the eigenvalue problem with the Rayleigh number as eigenvalue. Oscillatory convection cannot occur for the problem. The effects of Couple-stress parameter, Lewis number, modified diffusivity ratio, concentration Rayleigh number and porosity on stationary convection are shown both analytically and graphically.
\end{abstract}

KEY WORDS: Nanofluid, Couple-stress parameter, Darcy model, Brownian motion, Galerkin technique, perturbation method.

\section{INTRODUCTION}

The principle of thermal instability is an important phenomenon that has applications to different areas, such as geophysics, atmospheric physics, oceanography etc. The theoretical and experimental studies of thermal instability (Bénard convection) in a layer of fluid, under varying assumptions of hydrodynamics have been discussed in detail by Chandrasekhar [1]. The flow through porous medium has been of considerable interest in recent years, particularly in geophysics, soil sciences, ground water hydrology and astrophysics. However, the flow of a fluid through a homogeneous and isotropic porous medium is governed by Darcy's law, which states that the usual viscous term in the equations of fluid motion is replaced by the resistance term $-\frac{\mu}{k_{1}} \mathbf{q}$, where $\mu$ is viscosity of the fluid, $\mathrm{k}_{1}$ is permeability of medium and $\mathbf{q}$ is the Darcian (filter) velocity of the fluid. Lapwood [2] and Wooding [3] considered the stability

*Corresponding author e-mail: rameshnahan@yahoo.com 
of flow of a fluid through a porous medium, taking into account the Darcy's law. A detailed study of convection problems in a porous medium was also given by Ingham and Pop [4], Vafai and Hadim [5], and Nield and Bejan [6].

The presence of the nanoparticles in the fluid increased the effective thermal conductivity of the fluid and consequently enhanced the heat transfer characteristics. The term 'nanofluid' was first coined by Choi [7] and represents a significant class of heat transfer fluids, obtained by dispersing very small amount of nanoparticles in common base fluids. Nanoparticles, used in nanofluid are typically made of oxide ceramics $\left(\mathrm{Al}_{2} \mathrm{O}_{3}, \mathrm{CuO}\right)$, metal carbides $(\mathrm{SiC})$ or metals $(\mathrm{Al}, \mathrm{Cu})$ and base fluids are water, oil, bio-fluids, polymer solutions, other common fluids. The characteristic feature of nanofluid is the thermal conductivity enhancement, a phenomena observed by Masuda et al. [8]. Philip and Shima [9], Keblinski et al. [10], Wong and Leon [11], Yu and Xie [12], Taylor et al. [13] reported the developments in the study of heat transfer, using nanofluid.

Buongiorno [14] studied almost all aspects of the convective transport in nanofluids and developed a model for nanofluid, incorporating the effects of Brownian diffusion and thermophoresis. Using that model, Nield and Kuznetsov [15-17], Kuznetsov and Nield [18-20], Yadav et al. [21-22], Chand et al. [23, 24], Chand and Rana [2528], Chand [29], Umavathi and Mohite [30] and Rana et al. [31] studied the problems related to thermal instability in nanofluids. In all the above studies it was assumed that nanoparticle concentration can be imposed at the boundaries of the fluid. Recently, Nield and Kuznetsov [32], Chand and Rana [33-34], Chand et al. [35], Rana and Chand [36] pointed out that this type of boundary condition on volume fraction of nanoparticle is physically not realistic, as it is difficult to control the nanoparticle volume fraction on the boundaries and suggested the normal flux of volume fraction of nanoparticle is zero on the boundaries, as an alternative boundary condition, which is physically more realistic.

The above literature deals with the study of nanofluids as Newtonian nanofluid. The onset of convection in a horizontal layer of nanofluid as Newtonian nanofluid, uniformly heated from below (Bénard convection) has been extensively investigated, but a little attention has been made to study the thermal convection of non-Newtonian nanofluid. The investigations of such fluids are desirable with the growing importance of non-Newtonian nanofluids in technology and industries. In the category of non- Newtonian fluids, Couple-stress fluids have distinct features, such as polar effects. The theory of Couple-stress fluids has been formulated by Stokes [37]. One of the applications of Couple-stress fluids is the study of the lubrication mechanisms of synovial joints. A human joint is a dynamically loaded bearing, which has an articular cartilage as bearing and a synovial fluid as lubricant. Sharma and Thakur [38], Sharma and Sharma [39] considered the problem of a Couple-stress fluid, heated 
from below in a porous medium and reported that the Couple-stress parameter postponed the onset of stationary convection. Malashetty et al. [40] investigated the onset of convection in a Couple-stress fluid in a porous medium, using a thermal non-equilibrium model. Sunil et al. [41] studied global stability for thermal convection in a Couple-stress fluid and found that the linear and nonlinear stability Rayleigh number are the same.

Although thermal instability of non-Newtonian nanofluids problems was studied by Sheu [42], Chand and Rana [43] and Rana et al. [44], by taking different nonNewtonian as base fluid, but no effort has been put to investigate the thermal instability of Couple-stress nanofluid. Keeping in view the importance of Couple-stress nanofluid in a porous medium, an attempt has been made to study the thermal instability in a horizontal layer of Couple-stress nanofluid in a porous medium for more realistic boundary conditions.

\section{Mathematical FORMULATION OF THE PROBLEM}

We consider an infinite horizontal layer of Couple-stress nanofluid saturated porous layer, confined between the parallel boundaries $z=0$ and $z=d$, which are maintained at constant but different temperature $T_{0}$ at $z=0$ and $T_{1}$ at $z=d\left(T_{0}>T_{1}\right)$, as shown in Fig. 1.

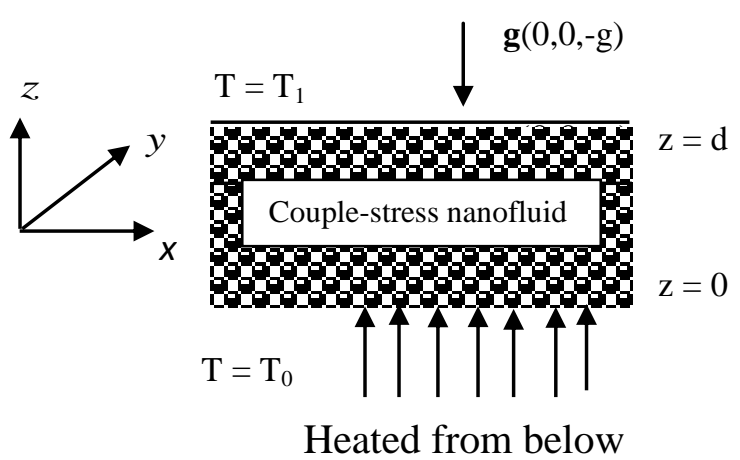

Fig. 1. Physical configuration of the problem

A Cartesian co-ordinate system $(x, y, z)$ is chosen, such that $z$ axis is taken at right angle to the boundaries and gravity $\mathbf{g}$ acts along the negative $z$ direction. The reference scale for temperature and nanoparticle fraction is taken to be $T_{1}$ and $\varphi_{0}$, respectively. 


\subsection{ASSUMPTIONS}

The mathematical equations, describing the physical model are based upon the following assumptions:

i. Thermophysical properties of fluid expect for density in the buoyancy force (Boussinesq Hypothesis) are constant,

ii. The fluid phase and nanoparticles are in thermal equilibrium state,

iii. Nanoparticles are spherical,

iv. No chemical reactions take place in fluid layer,

v. Size of nanoparticles is small as compared to pore size of the matrix,

vi. Nanoparticles are being suspended in the nanofluid, using either surfactant or surface charge technology, preventing the agglomeration and deposition of these on the porous matrix,

vii. Nanofluid is incompressible, Newtonian and laminar flow.

\subsection{GOVERNING EQUATIONS}

According to the works of Chandrasekhar [1], Nield and Kuznetsov [32] and Sharma and Thakur [38], the relevant basic equations for Couple-stress nanofluid in a porous medium under the Oberbeck- Boussinesq approximation are:

(1) $\nabla \cdot \mathbf{q}=0$

(2) $0=-\nabla p+\left(\varphi \rho_{p}+(1-\varphi)\left\{\rho_{f} 0\left(1-\alpha\left(T-T_{1}\right)\right)\right\}\right) \mathbf{g}-\frac{1}{k_{1}}\left(\mu-\mu_{c} \nabla^{2}\right) \mathbf{q}$,

(3) $(\rho c)_{m}\left(\frac{\partial T}{\partial t}+\mathbf{q} \cdot \nabla T\right)=k_{m} \nabla^{2} T+\varepsilon(\rho c)_{p}\left(D_{B} \nabla \varphi \cdot \nabla T+\frac{D_{T}}{T_{1}} \nabla T \cdot \nabla T\right)$,

(4) $\frac{\partial \varphi}{\partial t}+\frac{1}{\varepsilon} \mathbf{q} \cdot \nabla \varphi=D_{B} \nabla^{2} \varphi+\frac{D_{T}}{T_{1}} \nabla^{2} T$,

where $\mathbf{q}$ is the Darcian (filter) velocity, $p$ is the pressure, $\rho_{0}$ is the density of nanofluid at lower boundary, $\rho_{p}$ is the density of nanoparticle, $\varphi$ is the volume fraction of the nanoparticle, $T$ is the temperature, $\alpha$ is coefficient of the thermal expansion, $\mathbf{g}$ is acceleration due to gravity, $k_{1}$ is medium permeability of fluid, $\varepsilon$ is the porosity of porous medium, $\mu$ is the viscosity and $\mu_{c}$ is the Couple-stress viscosity, $(\rho c)_{m}$ is the heat capacity of fluid in porous medium, $(\rho c)_{p}$ is the heat capacity of nanoparticle, $k_{m}$ is the thermal conductivity of the fluid, $D_{B}$ is the Brownian diffusion coefficient, $D_{T}$ is the thermophoretic diffusion coefficient of the nanoparticle. 
We assume, that the temperature is constant and nanoparticle flux is zero on the boundaries. Thus, boundary conditions (Chandrasekhar [1] and Nield and Kuznetsov [32]) are:

$$
\begin{aligned}
& w=0, \quad T=T_{0}, \quad D_{B} \frac{\partial \phi}{\partial z}+\frac{D_{T}}{T_{1}} \frac{\partial T}{\partial z}=0 \text { at } z=0 \quad \text { and } \\
& w=0, \quad T=T_{1}, \quad D_{B} \frac{\partial \phi}{\partial z}+\frac{D_{T}}{T_{1}} \frac{\partial T}{\partial z}=0 \quad \text { at } z=d .
\end{aligned}
$$

Introducing non-dimensional variables, as:

$$
\begin{aligned}
& \left(x^{\prime}, y^{\prime}, z^{\prime}\right)=\left(\frac{x, y, z}{d}\right), \quad \mathbf{q}^{\prime}\left(u^{\prime}, v^{\prime}, w^{\prime}\right)=\mathbf{q}\left(\frac{u, v, w}{\kappa}\right) d, \\
& t^{\prime}=\frac{\kappa}{\sigma d^{2}} t, \quad p^{\prime}=\frac{k_{1}}{\mu \kappa} p, \quad \varphi^{\prime}=\frac{\left(\varphi-\varphi_{0}\right)}{\varphi_{0}}, \quad T^{\prime}=\frac{T}{\Delta T}
\end{aligned}
$$

where $\kappa=\frac{k_{m}}{(\rho c)_{f}}$ is the thermal diffusivity of the fluid, $\sigma=\frac{\left(\rho c_{p}\right)_{m}}{\left(\rho c_{p}\right)_{f}}$ is the thermal capacity ratio.

Equations (1) - (5) in non-dimensional form, can be written, as:

$$
\begin{aligned}
& \nabla \cdot \mathbf{q}=0 \\
& 0=-\nabla p-\left(1-\mathrm{C} \nabla^{2}\right) \mathbf{q}-\operatorname{Rm} \hat{e}_{z}+\operatorname{Ra} T \hat{e}_{z}-\operatorname{Rn} \varphi \hat{e}_{z}, \\
& \frac{\partial T}{\partial t}+\mathbf{q} \cdot \nabla T=\nabla^{2} T+\frac{N_{B}}{\mathrm{Le}} \nabla \varphi \cdot \nabla T+\frac{N_{A} N_{B}}{\mathrm{Le}} \nabla T \cdot \nabla T \\
& \frac{1}{\sigma} \frac{\partial \varphi}{\partial t}+\frac{1}{\varepsilon} \mathbf{q} \cdot \nabla \varphi=\frac{1}{\mathrm{Le}} \nabla^{2} \varphi+\frac{N_{A}}{\mathrm{Le}} \nabla^{2} T .
\end{aligned}
$$

[Primes ( ${ }^{\prime}$ ) have been dropped for simplicity]

Here, the non-dimensional parameters are given, as:

$$
\begin{aligned}
& \mathrm{Le}=\frac{\kappa}{D_{B}} \text { is the Lewis number, } \\
& \mathrm{Ra}=\frac{\rho_{0} \alpha g k_{1} d\left(T_{0}-T_{1}\right)}{\mu \kappa} \text { is the Rayleigh-Darcy number, } \\
& \mathrm{Rm}=\frac{\left(\rho_{p} \varphi_{0}+\rho_{0}\left(1-\varphi_{0}\right)\right) g k_{1} d}{\mu \kappa} \text { is the basic-density Rayleigh number, } \\
& \mathrm{Rn}=\frac{\left(\rho_{p}-\rho\right) \varphi_{0} g k_{1} d}{\mu \kappa} \text { is the nanoparticle concentration Rayleigh number, }
\end{aligned}
$$




$$
\begin{aligned}
& \mathrm{C}=\frac{\mu_{c}}{\mu d^{2}} \text { is the Couple-stress parameter, } \\
& N_{A}=\frac{D_{T}\left(T_{0}-T_{1}\right)}{D_{B} T_{1} \varphi_{0}} \text { is the modified diffusivity ratio, } \\
& N_{B}=\frac{\varepsilon(\rho c)_{p} \varphi_{0}}{(\rho c)_{f}} \text { is the modified particle-density increment. }
\end{aligned}
$$

The dimensionless boundary conditions are:

$$
\begin{array}{ll}
w=0, & T=1, \quad \frac{\partial \phi}{\partial z}+N_{A} \frac{\partial T}{\partial z}=0 \text { at } z=0 \text { and } \\
w=0, & T=0, \quad \frac{\partial \phi}{\partial z}+N_{A} \frac{\partial T}{\partial z}=0 \text { at } z=1 .
\end{array}
$$

\subsection{BASIC SOLUTIONS}

The basic state was assumed to be quiescent and is given by:

$$
u=v=w=0, \quad p=p(z), \quad T=T_{b}(z) \quad \varphi=\varphi_{b}(z) .
$$

Equations (6) - (9), using boundary conditions (10) give solution as:

$$
T_{b}=1-z, \quad \varphi_{b}=\phi_{0}+N_{A} z
$$

where $\varphi_{0}$ is reference value for nanoparticle volume fraction. The basic solution for temperature and nanoparticle volume fraction is identical with solution obtained by Nield and Kuznetsov [32].

\subsection{Perturbation SOlutions}

To study the stability of the system, we superimposed infinitesimal perturbations on the basic state, which are written in following forms:

(11) $q(u, v, w)=0+q^{\prime \prime}(u, v, w), \quad T=T_{b}+T^{\prime \prime}, \quad \varphi=\varphi_{b}+\varphi^{\prime \prime}, \quad p=p_{b}+p^{\prime \prime}$,

with

$$
T_{b}=1-z, \quad \varphi_{b}=\phi_{0}+N_{A} z .
$$

Using equation (11) in equations (6) - (9) and by neglecting the product of the prime quantities, we obtain the following equations: 


$$
\begin{aligned}
& \nabla \cdot \mathbf{q}=0, \\
& 0=-\nabla p-\left(1-\mathrm{C} \nabla^{2}\right) \mathbf{q}+\operatorname{RaT} \hat{e}_{z}-\operatorname{Rn} \varphi \hat{e}_{z} \\
& \frac{\partial T}{\partial t}-w=\nabla^{2} T+\frac{N_{B}}{\mathrm{Le}}\left(N_{A} \frac{\partial T}{\partial z}-\frac{\partial \varphi}{\partial z}\right)-\frac{2 N_{A} N_{B}}{\mathrm{Le}} \frac{\partial T}{\partial z}, \\
& \frac{1}{\sigma} \frac{\partial \varphi}{\partial t}+\frac{N_{A}}{\varepsilon} w=\frac{1}{\mathrm{Le}} \nabla^{2} \varphi+\frac{N_{A}}{\mathrm{Le}} \nabla^{2} T .
\end{aligned}
$$

[Double primes ( $\left.{ }^{\prime \prime}\right)$ have been dropped for simplicity]

Eliminating pressure term ' $p$ ' from equation (13), we have:

$$
\left(1-\mathrm{C} \nabla^{2}\right) \nabla^{2} w=\operatorname{Ra} \nabla_{H}^{2} T-\operatorname{Rn} \nabla_{H}^{2} \varphi
$$

where $\nabla_{H}^{2}=\frac{\partial^{2}}{\partial x^{2}}+\frac{\partial^{2}}{\partial y^{2}}$ is the two-dimensional Laplacian operator on the horizontal plane.

Boundary conditions are:

$$
w=0, \quad T=0, \quad \frac{\partial \phi}{\partial z}+N_{A} \frac{\partial T}{\partial z}=0 \text { at } z=0,1 .
$$

\section{NORMAL MODE ANALYSIS}

Analyzing the disturbances into the normal modes and assuming, that the perturbed quantities are of the form:

$$
[w, T, \phi]=[W(z), \Theta(z), \Phi(z)] \exp \left(i k_{x} x+i k_{y} y+n t\right),
$$

where $k_{x}$ and $k_{y}$ are wave numbers in $x$ and $y$ directions respectively, while n (complex constant) is the growth rate of disturbances.

Using equation (18), equations (16), (14) - (15) become:

$$
\begin{aligned}
& \left(1-\mathrm{C}\left(D^{2}-a^{2}\right)\right)\left(D^{2}-a^{2}\right) W+a^{2} \operatorname{Ra} \Theta-a^{2} \operatorname{Rn} \Phi=0, \\
& W+\left(D^{2}-a^{2}-n+\frac{N_{A}}{\mathrm{Le}} D-\frac{2 N_{A} N_{B}}{\mathrm{Le}} D\right) \Theta-\frac{N_{B}}{\mathrm{Le}} D \Phi=0, \\
& \frac{W}{\varepsilon}-\frac{N_{A}}{\mathrm{Le}}\left(D^{2}-a^{2}\right) \Theta-\left(\frac{1}{\mathrm{Le}}\left(D^{2}-a^{2}\right)-\frac{n}{\sigma}\right) \Phi=0,
\end{aligned}
$$

where $D \equiv \frac{d}{d z}$ and $a=\sqrt{k_{x}^{2}+k_{y}^{2}}$ is the dimensionless resultant wave number.

The boundary conditions of the problem, in view of normal mode analysis are:

$$
W=0, \quad \Theta=0, \quad D \Phi+N_{A} D \Theta=0 \text { at } z=0,1 .
$$




\section{Method of SOlution}

The Galerkin weighted residuals method is used to obtain an approximate solution to the system of equations (19) - (21) with the boundary conditions (22). In this method, the test functions are the same as the base (trial) functions. Accordingly $W$, $\Theta$ and $\Phi$ are taken as:

$$
W=\sum_{p=1}^{N} A_{p} W_{p}, \quad \Theta=\sum_{p=1}^{N} B_{p} \Theta_{p}, \quad \Phi=\sum_{p=1}^{N} C_{p} \Phi_{p},
$$

where $A_{p}, B_{p}$ and $C_{p}$ are unknown coefficients, $p=1,2,3, \ldots, N$ and the base functions $W_{p}, \Theta_{p}$, and $\Phi_{p}$, satisfying the boundary conditions (22). Using expression for $W, \Theta$ and $\Phi$ in equations (19) - (21) and multiplying the first equation by $W_{p}$, the second equation by $\Theta_{p}$, third equation by $\Phi_{p}$ and then integrating in the limits from zero to unity, we obtain a set of $3 N$ linear homogeneous equations with $3 N$ unknown $A_{p}, B_{p}$ and $C_{p} ; p=1,2,3, \ldots, N$. For existing of non trivial solution, the vanishing of the determinant of coefficients produces the characteristics equation of the system in term of Rayleigh number Ra.

\section{LINEAR STABILITY ANALYSIS}

Oscillatory convection is ruled out, because of the absence of the two opposing buoyancy forces, so we consider the case of the stationary convection. For the first Galerkin approximation, we take $N=1$; the appropriate trial function (Nield and Kuznetsov [32]) satisfying boundary condition (22) is given by:

$$
W_{1}=\Theta_{1}=z(1-z), \quad \Phi_{1}=-N_{A} z(1-z) .
$$

Substituting trail functions (24) in the system of equations (19) - (21) and using boundary condition (22), we obtain the eigenvalue equation for stationary convection $(n=0)$, as:

$$
\mathrm{Ra}=\frac{\left(a^{2}+10\right)^{2}+\mathrm{C}\left(a^{2}+10\right)\left(a^{4}+20 a^{2}+120\right)}{a^{2}}-\left(1+\frac{\mathrm{Le}}{\varepsilon}\right) N_{A} \mathrm{Rn} .
$$

The minimum value of the Rayleigh number Ra occurs at the critical wave number $a=a_{c}$, where $a_{c}$ satisfies the equation:

$$
2 \mathrm{C}\left(a_{c}^{2}\right)^{3}+(300 \mathrm{C}+1)\left(a_{c}^{2}\right)^{2}-(1200 \mathrm{C}+100)=0 .
$$

It is important to note, that the critical wave number $a_{c}$ depends on the couplestress parameter $\mathrm{C}$. In the absence of Couple-stress parameter $(\mathrm{C}=0)$, minimum 
value of the Rayleigh number $\operatorname{Ra}_{c}$ occurs at $a=\sqrt{10}$ and is given by:

$$
\mathrm{Ra}_{c}=40-\left(1+\frac{\mathrm{Le}}{\varepsilon}\right) N_{A} \mathrm{Rn} .
$$

This is exactly the same result which was obtained by Nield and Kuznetsov [32].

In the absence of both Couple-stress parameter and nanoparticles $(\mathrm{C}=0, \mathrm{Rn}=$ 0 ), critical value of the Rayleigh number is given by:

$$
\mathrm{Ra}_{c}=40,
$$

which is approximately equal to the classical results, obtained by Horton and Rogers [45] and Lapwood [2].

In order to investigate the effects of the Couple-stress parameter $\mathrm{C}$, Lewis number Le, modified diffusivity ratio $N_{A}$, nanoparticle concentration, Rayleigh number $\mathrm{Rn}$ and porosity parameter $\varepsilon$ on stationary convection, we examine the behaviour of $\frac{\partial \mathrm{Ra}}{\partial \mathrm{C}}, \frac{\partial \mathrm{Ra}}{\partial \mathrm{Le}}, \frac{\partial \mathrm{Ra}}{\partial N_{A}}, \frac{\partial \mathrm{Ra}}{\partial \mathrm{Rn}}$ and $\frac{\partial \mathrm{Ra}}{\partial \varepsilon}$ analytically. From equation (25), we have:

$$
\begin{gathered}
\frac{\partial \mathrm{Ra}}{\partial \mathrm{C}}>0, \quad \frac{\partial \mathrm{Ra}}{\partial \varepsilon}>0 \quad \text { and } \\
\frac{\partial \mathrm{Ra}}{\partial \mathrm{Le}}<0, \quad \frac{\partial \mathrm{Ra}}{\partial N_{A}}<0, \quad \frac{\partial \mathrm{Ra}}{\partial \mathrm{Rn}}<0 .
\end{gathered}
$$

These inequalities shows that Couple-stress parameter and porosity parameter have stabilizing effect while Lewis number, modified diffusivity ratio and nanoparticle concentration Rayleigh number have destabilizing effect on the stationary convection.

\section{RESULT AND DISCUSSION}

Thermal instability in a horizontal layer of Couple-stress nanofluid in a porous medium is investigated for more realistic boundary conditions. Equation (25) expresses the thermal stationary Rayleigh number Ra, as a function of dimensionless wave number ' $a$ ' and Couple-stress parameter $\mathrm{C}$, Lewis number Le, modified diffusivity ratio $N_{A}$, nanoparticle concentration Rayleigh number Rn and porosity parameter $\varepsilon$. It is also noted, that parameter $N_{B}$ does not appear in the equation, thus, instability is purely phenomenon, due to buoyancy coupled with the conservation of nanoparticle. It is independent on the contributions of Brownian motion and thermophoresis to the thermal energy equation. The parameter $N_{B}$ drops out, because of an orthogonal property of the first order trail functions and their first derivatives. 
Numerical computations are carried out for different values of Couple-stress parameter C, Lewis number Le, modified diffusivity ratio $N_{A}$ and nanoparticle concentration Rayleigh number Rn. As per Nield and Kuznetsov [32], Chand [46], Sharma and Sharma [39], the parameters considered are in the range of $10^{2} \leq \mathrm{Ra} \leq 10^{4}$ (thermal Rayleigh number), $1 \leq \mathrm{C} \leq 10^{2}$ (Couple-stress parameter), $10^{2} \leq \mathrm{Le} \leq 10^{4}$ (nanofluid Lewis number), $1<N_{A}<10$ (modified diffusivity ratio) and $1 \leq \mathrm{Rn} \leq$ 10 (nanoparticle concentration Rayleigh number).

Stability curve for Couple-stress parameter C, Lewis number Le, modified diffusivity ratio $N_{A}$ and nanoparticle concentration Rayleigh number $\mathrm{Rn}$ are depicted in figures 2-6.

Figure 2 shows the neutral stability curves for different values of the Couple-stress parameter and fixed values of other parameters. We observe from this figure, that the minimum value of the Rayleigh number increases with an increase in the value of the couple-stress parameter $\mathrm{C}$, indicating that the effect of the Couple-stress parameter is to stabilize the system. This is good agreement of result, obtained by Sharma and Thakur [38] and Sharma and Sharma [39].

The effect of Lewis number Le on the neutral stability curves for fixed values of other parameters is shown in Fig. 3. We find, that the minimum value of Rayleigh number decreases with an increase in the value of Lewis number Le. Thus, Lewis number has a destabilizing effect on the system. This is the good agreement of the result, obtained by Chand and Rana [33-34].

Figure 4 displays the effect of the modified diffusivity ratio on the neutral stability curves, for fixed values of other parameters. This figure indicates that the minimum

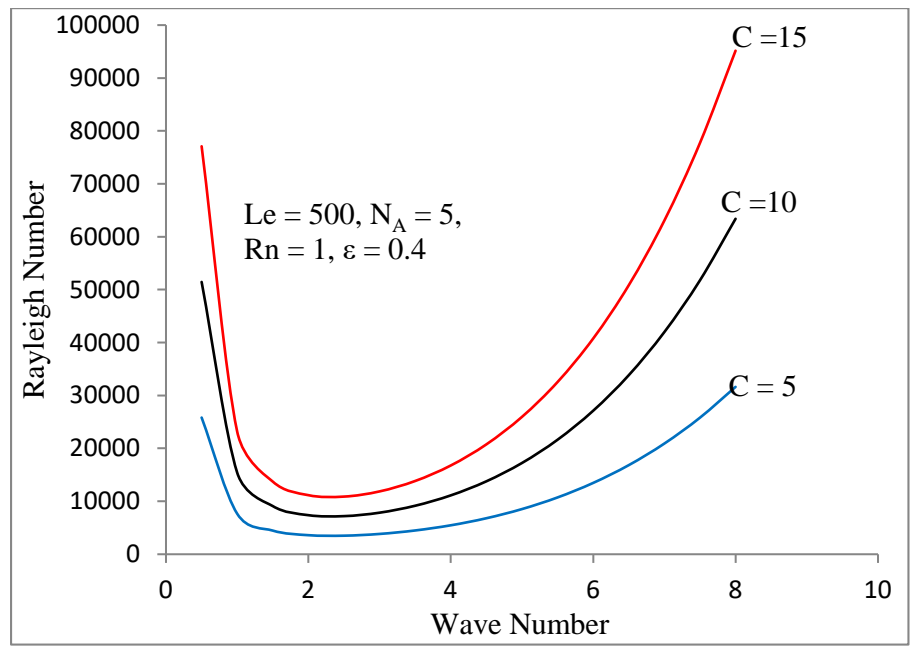

Fig. 2. Neutral stability curves for different value of the Couple-stress parameter. 


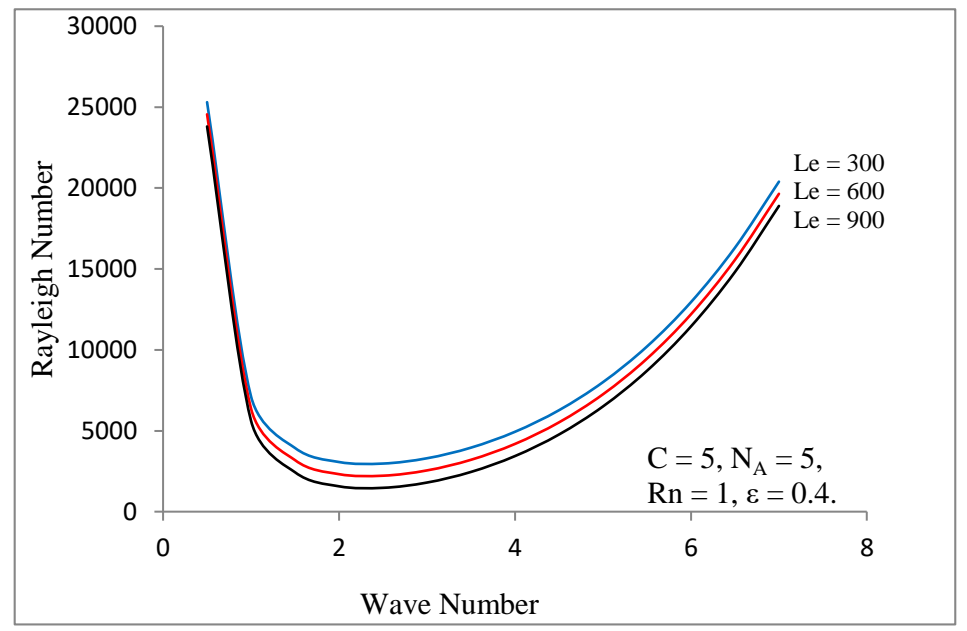

Fig. 3. Neutral stability curves for different value of the Lewis number.

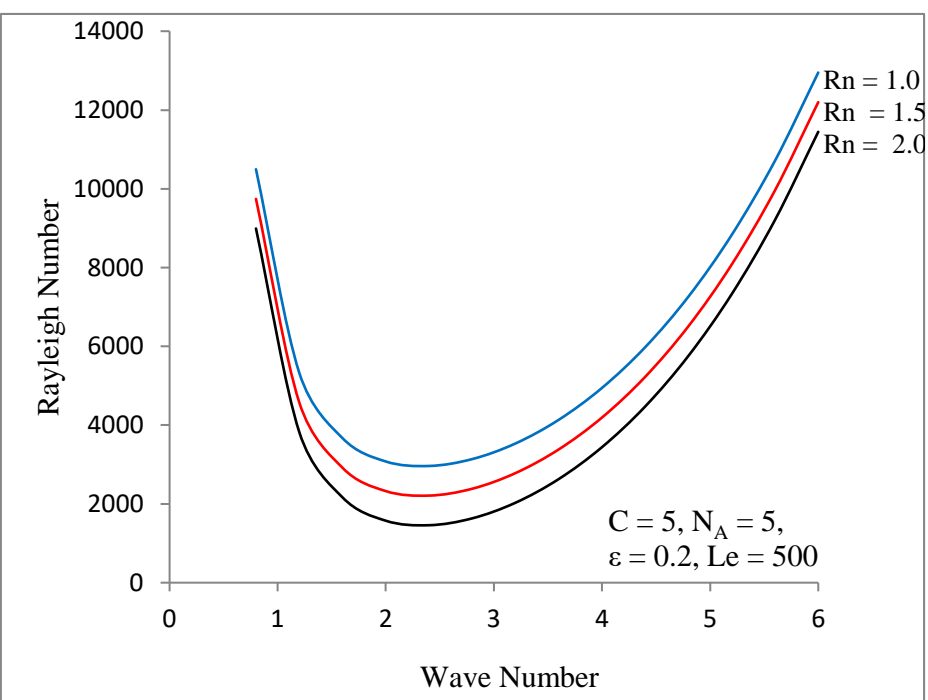

Fig. 4. Neutral stability curves for different value of a nanoparticle concentration Rayleigh number.

Rayleigh number slightly decreases with an increase in the value of the modified diffusivity ratio, indicating a destabilizing effect of the modified diffusivity ratio on fluid layer. This is the good agreement of the result, obtained by Chand and Rana [33-34]. 


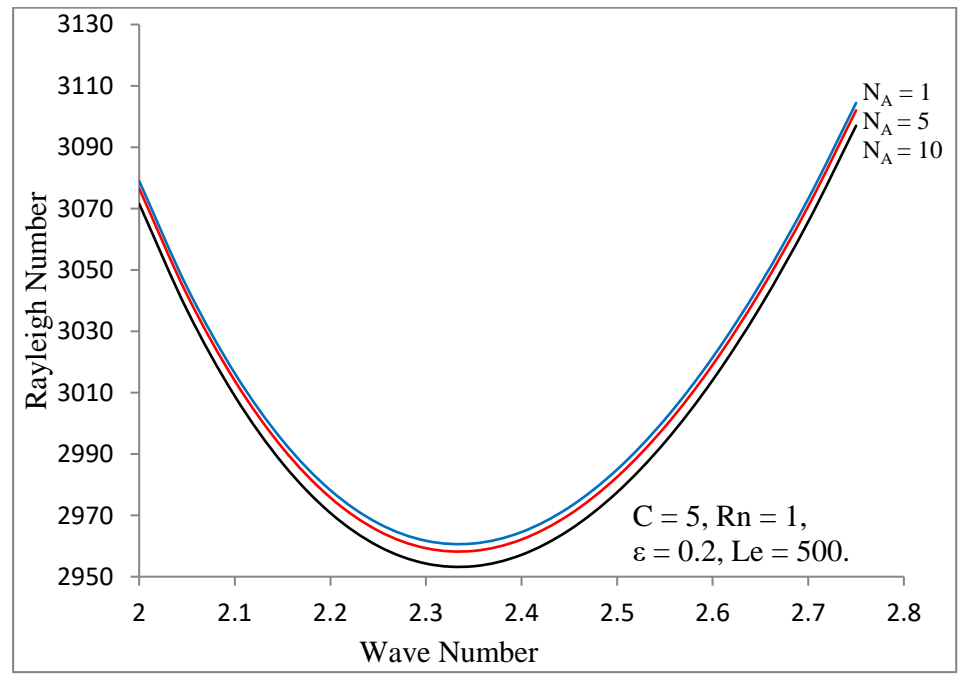

Fig. 5. Neutral stability curves for different value of the modified diffusivity ratio.

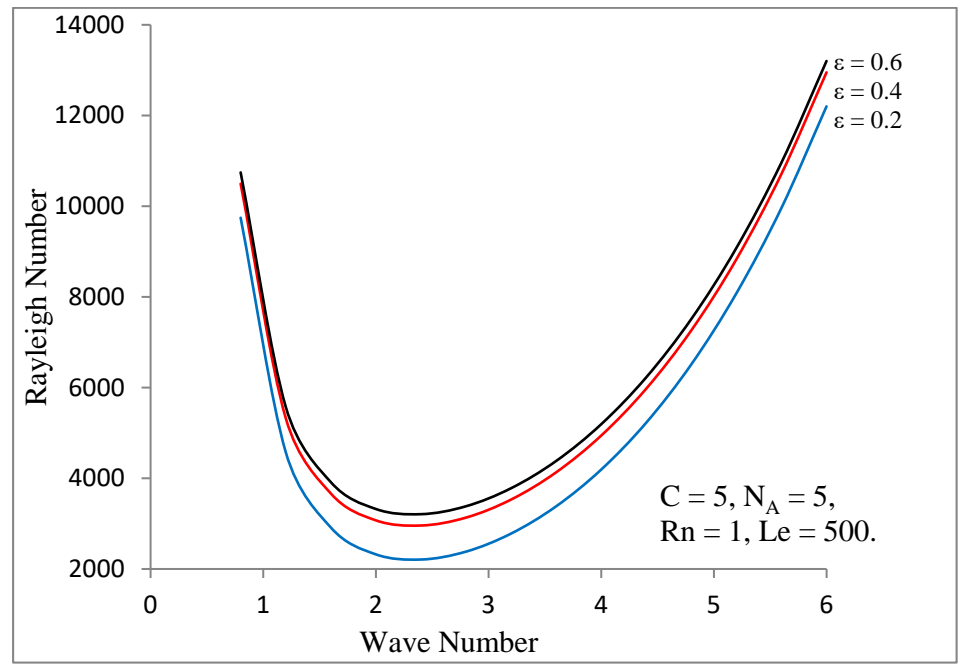

Fig. 6. Neutral stability curves for different value of the porosity parameter.

The effect of nanoparticle concentration Rayleigh number Rn on neutral curves, is shown in Fig. 5 for fixed values of other parameters. It is observed, that Rayleigh number decreases as the value of nanoparticle concentration Rayleigh number increases, indicating that the nanoparticle concentration Rayleigh number destabilizes the system. This is good agreement of result obtained by Nield and Kuznetsov [32], Chand and Rana [33-34]. 
Figure 6 shows the neutral stability curves for different values of the porosity parameter and fixed values of other parameters. We observe from this figure, that the minimum value of the Rayleigh number increases with an increase in the value of the porosity parameter, indicating that the effect of the porosity parameter is to stabilize the system.

\section{Conclusions}

Thermal instability of a Couple-stress nanofluid in a porous medium is investigated theoretically for more realistic boundary conditions. The model used for nanofluid incorporates the effect of Brownian diffusion and thermophoresis. The flux of volume fraction of nanoparticle is taken to be zero on the isothermal boundaries. The eigenvalue problem is solved numerically by using the Galerkin technique with the Rayleigh number as eigenvalue. The effects of Couple- stress parameter $\mathrm{C}$, Lewis number Le, modified diffusivity ratio $N_{A}$, nanoparticle concentration Rayleigh number $\mathrm{Rn}$ and porosity parameter $\varepsilon$ on stationary convection have been presented both analytically and graphically.

The main conclusions of present analysis are, as follows:

1. The instability purely phenomenon, due to buoyancy coupled with the conservation of nanoparticle and is independent on the contribution of Brownian motion and thermophoresis.

2. Oscillatory convection cannot occur for the problem.

3. The critical value of the Rayleigh number depends upon the Couple-stress.

4. The couple-stress parameter and porosity parameter have stabilizing effect, while the Lewis number, modified diffusivity ratio and nanoparticle concentration Rayleigh number have destabilizing effect on stationary convection.

\section{ACKNOWLEDGMENT}

The authors are grateful to the reviewers for their valuable comments and suggestions for improvement of the paper.

\section{REFERENCES}

[1] Chandrasekhar, S. Hydrodynamic and Hydromagnetic Stability, New York, Oxford University Press Dover Publication, 1981.

[2] Lapwood, E. R. Convection of a Fluid in Porous Medium. Proc. Camb. Phil. Soc., 44 (1948), 508-519.

[3] Wooding, R. A. Rayleigh Instability of a Thermal Boundary Layer an Flow Through a Porous Medium. J. Fluid Mech., 9 (1960), 183-192. 
[4] Ingham, D. D., L. Pop. Transport Phenomena in Porous Media, New York, Elsvier, 1981.

[5] VAfai, K. A., H. A. Hadim. Hand Book of Porous Media, New York, M. Decker, 2000.

[6] Nield, D. A., A. Bejan. Convection in Porous Medium, New York, Springer, 2013.

[7] CHOI, S. Enhancing Thermal Conductivity of Fluids with Nanoparticles. In: Siginer DA, Wang HP, Editors. Developments and Applications of Non-Newtonian Flows. American Society of Mechanical Engineers, 66 (1995), 99-105.

[8] Masuda, H., A. Ebata, K. Teramae, N. Hishinuma. Alternation of Thermal Conductivity and Viscosity of Liquid by Dispersing Ultra-Fine Particles (Dispersion of- $\mathrm{Al}_{2} \mathrm{O}_{3}, \mathrm{SiO}_{2}$ and $\mathrm{TiO}_{2}$ Ultra-Fine Particles). Netsu Bussei (Japan), 4 (1993), 227 233.

[9] Philip, J., P. D. Shima. Thermal Properties of Nanofluids. Adv., Coll. and Interface Science, 15 (2012), 30-45.

[10] Keblinski, P., L. W. Hu, J. L. Alvarado. A Benchmark Study on Thermal Conductivity of Nanofluid. J. Appl. Phys., 106 (2009), 094312.

[11] Wong, K. V., O. De. LeOn. Applications of Nanofluids: Current and Future. Advances in Mechanical Engineering, 2010 (2010), 1-11.

[12] Yu, W., H. XIE. A Review on Nanofluids: Preparation, Stability Mechanisms and Applications. J. of Nanomaterials, 2012 (2012), 1-17.

[13] Taylor, R., S. Coulombe, T. Otanicar, P. Phelan, A. Gunawan, W. Lv, G. Rosengarten, R. Prashar, H. Tyagi. Small Particles, Big Impacts: A review of the Diverse Applications of Nanofluids. J. Appl. Phys., 113 (2013), 011301.

[14] Buongiorno, J. Convective Transport in Nanofluids. ASME Journal of Heat Transfer, 128 (2006), 240-250.

[15] Nield, D. A., A. V. Kuznetsov. Thermal Instability in a Porous Medium Layer Saturated by A Nanofluid. Int. J. Heat Mass Trans, 52 (2009), 5796-5801.

[16] Nield, D. A., A. V. Kuznetsov. The Onset of Convection in a Layer of Cellular Porous Material: Effect of Temperature-Dependent Conductivity Arising From Radiative Transfer. J. Heat Trans., 132 (2010), No. 7, 074503-074504.

[17] Nield, D. A., A. V. Kuznetsov. The Effect of Vertical through Flow on Thermal Instability in a Porous Medium Layer Saturated by a Nanofluid. Trans. Porous Media, 87 (2011), 765-775.

[18] Kuznetsov, A. V., D. A. Nield. Effect of Local Thermal Non-Equilibrium on the Onset of Convection in a Porous Medium Layer Saturated by a Nanofluid. Transp. Porous Media, 83 (2010), 425-436.

[19] Kuznetsov, A. V., D. A. Nield. Thermal Instability in a Porous Medium Layer Saturated by a Nanofluid: Brinkman Model. Trans. Porous Medium, 81 (2010), 409422.

[20] Kuznetsov, A. V., D. A. Nield. The Onset of Double-Diffusive Nanofluid Convection in a Layer of a Saturated Porous Medium. Trans. Porous Media, 85 (2010), No. 3, 941-951. 
[21] YadaV, D., G. S. Agrawal, R. BhargaVA. Thermal Instability of Rotating Nanofluid Layer. International Journal of Engineering Science, 49 (2011), 1171-1184.

[22] Yadav, D., G. S. Agrawal, R. Bhargava. The Onset of Convection in a Binary Nanofluid Saturated Porous Layer. Int. J. of Theoretical and Applied Multiscale Mechanics, 2 (2012), No. 3, 198-224

[23] Chand, R., G. C. RAnA, S. Kumar. Variable Gravity Effects on Thermal Instability of Nanofluid in Anisotropic Porous Medium. Int. J. of Appl. Mech. and Engg., 18 (2013), No. 3, 631-642.

[24] Chand, R., G. C. RanA, A. K. Hussein. On The Onset of Thermal Instability in a Low Prandtl Number Nanofluid Layer in a Porous Medium. Journal of Applied Fluid Mechanics, 8 (2015), No. 2, 265-272.

[25] Chand, R., G. C. RANA. On The Onset of Thermal Convection in Rotating Nanofluid Layer Saturating a Darcy-Brinkman Porous Medium. Int. J. of Heat and Mass Transfer, 55 (2012), 5417-5424.

[26] Chand, R., G. C. RanA. Oscillating Convection of Nanofluid in Porous Medium. Transp. Porous Med., 95 (2012), 269-284.

[27] Chand, R., G. C. RANA. Hall Effect on the Thermal Instability in a Horizontal Layer of Nanofluid. Journal of Nanofluids, 3 (2014), 247-253.

[28] Chand, R., G. C. RanA. Hall Effect on Thermal Instability in a Horizontal Layer of Nanofluid Saturated in a Porous Medium. Int. J. of Theoretical and Applied Multiscale Mechanics, 3, (2014), No. 1, 58-73.

[29] Chand, R. On the Onset of Rayleigh-Bénard Convection in a Layer of Nanofluid in Hydromagnetics. Int. J. of Nanoscience, 12 (2013), No. 6, 1350038.

[30] Umavathi, J. C., M. B. Monite. The Onset of Convection in a Nanofluid Saturated Porous Layer Using Darcy Model With Cross Diffusion. Meccanica, 49 (2014), No. 5, 1159-1175.

[31] Rana, G. C., R. C. Thakur, S. K. Kango. On the Onset of Double-Diffusive Convection in a Layer of Nanofluid under Rotation Saturating a Porous Medium. Journal of Porous Media, 17 (2014), No. 8, 657-667.

[32] Nield, D. A., A. V. Kuznetsov. Thermal Instability in a Porous Medium Layer Saturated by a Nanofluid: A Revised Model. Int. J. of Heat and Mass Transfer, 68 (2014), No. 4, 211-214.

[33] Chand, R., G. C. Rana. Magneto Convection in a Layer of Nanofluid in Porous Medium-A More Realistic Approach. Journal of Nanofluids, 4 (2015), 196-202.

[34] Chand, R., G. C. RANA. Thermal Instability in a Brinkman Porous Medium Saturated by Nanofluid With No Nanoparticle Flux on Boundaries. Special Topics \& Reviews in Porous Media: An International Journal, 5 (2014), No. 4, 277-286.

[35] Chand, R., R. Kango, G. C. Rana. Thermal Instability in Anisotropic Porous Medium Saturated by a Nanofluid-A Realistic Approach. NSNTAIJ, 8 (2014), No. 12, 445-453. 
[36] Rana, G. C., R. Chand. On the Thermal Convection in a Rotating Nanofluid Layer Saturating a Darcy-Brinkman Porous Medium: A More Realistic Model. Journal of Porous Media, 18 (2015), No. 6, 629-635.

[37] Stokes, V. K. Couple Stresses in Fluids. Phys. Fluids, 9 (1966), 1709-1716.

[38] Sharma, R. C., K. D. Thakur. On Couple Stress Fluid Heated From Below in Porous Medium in Hydrodynamics. Czech. J. Phys., 50 (2000), 753-758.

[39] Sharma, R. C., M. Sharma. Effect of Suspended Particles on Couple-Stress Fluid Heated From Below in the Presence of Rotation and Magnetic Field. Ind. J. Pure Appl. Math., 35 (2004), 973-989.

[40] Malashetty, M. S., I. S. Shivakumar, S. Kulkarni. The Onset of Convection in Couple Stress Fluid Saturated Porous Layer Using a Thermal Non-Equilibrium Model. Phys. Lett. A, 373 (2009), 781-790.

[41] Sunil, D. R., A. Mahajan. Global Stability for Thermal Convection in a CoupleStress Fluid. International Communications in Heat and Mass Transfer, 38 (2011), 938-942.

[42] Sheu, L. J. Thermal Instability in a Porous Medium Layer Saturated with a ViscoElastic Nanofluid. Transp Porous Med, 88 (2011), 461- 477.

[43] Chand, R., G. C. Rana. Thermal Instability of Rivlin-Ericksen Elastico-Viscous Nanofluid Saturated by a Porous Medium. J. Fluids Eng., 134 (2012), No. 12, 121203.

[44] Rana, G. C., R. C. ThaKur, S. K. Kango. On the Onset of Thermosolutal Instability in a Layer of an Elastico-Viscous Nanofluid in Porous Medium. FME Transactions, 42 (2014), 1-9.

[45] Horton, C. W., F. T. Rogers. Convection Currents in a Porous Medium. J. Appl. Phys. 16 (1945), 367-70.

[46] Chand, R. Nanofluid Technologies and Thermal Convection Techniques. IGI Global, (2017). doi:10.4018/978-1-68318-006-7. 